\title{
Revealing the Absolute Direction of the Dzyaloshinskii-Moriya Interaction in Prototypical Weak Ferromagnets by Polarized Neutrons
}

\author{
H. Thoma $\odot,{ }^{1, *}$ V. Hutanu $\odot,{ }^{2, \dagger}$ H. Deng $\odot,{ }^{2, \ddagger}$ V. E. Dmitrienko, ${ }^{3}$ P. J. Brown, ${ }^{4}$ A. Gukasov $\odot,{ }^{5}$ G. Roth, ${ }^{6}$ and M. Angst $\odot^{7, \S}$ \\ ${ }^{1}$ Jülich Centre for Neutron Science (JCNS) at Heinz, Maier-Leibnitz Zentrum (MLZ), \\ Forschungszentrum Jülich GmbH, 85748 Garching, Germany \\ ${ }^{2}$ Institute of Crystallography, RWTH Aachen University and Jülich Centre for Neutron Science (JCNS) at \\ Heinz Maier-Leibnitz Zentrum (MLZ), 85748 Garching, Germany \\ ${ }^{3}$ A. V. Shubnikov Institute of Crystallography, FRSC “Crystallography and Photonics," RAS, \\ Moscow 119333, Russia \\ ${ }^{4} 12$ Little St Marys Lane, Cambridge, CB2 1RR, United Kingdom \\ ${ }^{5}$ Laboratoire Léon Brillouin, CEA-CNRS, CE-Saclay, 91191 Gif-sur-Yvette, France \\ ${ }^{6}$ Institute of Crystallography, RWTH Aachen University, 52056 Aachen, Germany \\ ${ }^{7}$ Jülich Centre for Neutron Science JCNS and Peter Grünberg Institut PGI, \\ JARA-FIT, Forschungszentrum Jülich GmbH, 52425 Jülich, Germany
}

(Received 9 June 2020; revised 29 November 2020; accepted 14 January 2021; published 25 March 2021)

\begin{abstract}
Polarized neutron diffraction (PND) is a powerful technique to distinguish a weak magnetic contribution from the total scattering intensity. It can provide a detailed insight into the microscopic spin ordering at the unit cell level, but also into the mesoscopic magnetic ordering, like different types of domain populations. Here we report on the application of this technique to the long-standing problem of determining the absolute direction of the Dzyaloshinskii-Moriya vector in relation to the crystal structure. The proposed PND method, based on the measurement of one representative reflection, is easy to perform and straightforward to interpret. The absolute sign of the Dzyaloshinskii-Moriya interaction (DMI) in $\mathrm{MnCO}_{3}$ has been independently determined by PND and found to be in agreement with recent results obtained by resonant magnetic synchrotron scattering. This validates the method. In addition, the absolute DMI vector direction in the prototypical room-temperature weak ferromagnet $\alpha-\mathrm{Fe}_{2} \mathrm{O}_{3}$ (hematite) has been determined for the first time. To demonstrate the generality of our method, further examples with different symmetries are also presented. Ab initio calculations of the resulting weak noncollinear magnetization using the QUANTUM ESPRESSO package, considering DMI in addition to the symmetric magnetic exchange interaction, were also conducted and found to be in agreement with the experimental results from PND.
\end{abstract}

DOI: 10.1103/PhysRevX.11.011060

\section{INTRODUCTION}

The Dzyaloshinskii-Moriya interaction (DMI), introduced in the late 1950s to explain the small canting of magnetic moments away from the perfect collinear antiferromagnetic (AFM) alignment [1-4], became of particular interest in current condensed matter research as it is present in a wide range of complex magnetic materials, such as multiferroics [5,6], topological insulators [7], and

\footnotetext{
*h.thoma@fz-juelich.de

†ladimir.hutanu@frm2.tum.de

*hao.deng@frm2.tum.de

${ }^{\S}$ m.angst@fz-juelich.de
}

Published by the American Physical Society under the terms of the Creative Commons Attribution 4.0 International license. Further distribution of this work must maintain attribution to the author(s) and the published article's title, journal citation, and DOI.
Subject Areas: Condensed Matter Physics,

Magnetism, Spintronics antiferromagnetic spin-wave field-effect transistors [8], and is the driving force to stabilize various novel topological noncollinear magnetic structures, such as spin spirals [9], magnetic skyrmions [10], or magnetic soliton lattices [11]. The DMI is commonly attributed to relativistic spin-orbit coupling in conjunction with a broken inversion symmetry [1-4]. Based on the type of inversion symmetry breaking, the DMI can be classified into the bulk and the interfacial DMI, with the former arising from a local inversion symmetry breaking in the atomic structure whereas the latter is caused by a lack of inversion symmetry only at a layer interface [9].

Compared to the symmetric exchange energy $\propto S_{1} \cdot S_{2}$, the magnitude of the antisymmetric DMI, scaling with the vector product $S_{1} \times S_{2}$ of the two spins, is usually small, but its direction, as emphasized by $\mathrm{Hu}$ [12], is often a decisive factor in determining the system's chirality for both bulk and interfaces, but it is particularly crucial for thin film systems [13]. For example, in ultrathin magnetic 
heterostructures with Néel-like domain wall spirals, the DMI sign is directly coupled to the direction to which a domain wall moves with current via the spin Hall torque $[14,15]$. Thus, understanding the underlying physics to correctly predict the sign of the DMI and to optimize the current-driven domain wall motion is a key step toward the development of novel advanced nonvolatile memory devices [16]. In addition, the DMI sign has a fundamental impact on skyrmions, topologically stable magnetic structures that are promising candidates as information carriers for future ultrahigh-density storage and logic devices [13,17-21]. By interface engineering, i.e., sandwiching the skyrmion carrying magnetic layer between two heavymetal films characterized by interfacial DMI constants of opposite sign, a strong DMI was achieved to facilitate ultradense skyrmion packaging [22]. Furthermore, to realize a straight, longitudinal transmission of isolated magnetic skyrmions in thin films, which is essential for future skyrmionic devices, the skyrmion Hall effect, leading to a transverse motion, must be suppressed [23]. A recent approach for such a suppression is the mixture of interfacial and bulk DMIs, causing an asymmetry in the spin-orbittorque-induced skyrmion Hall angle for the two different skyrmion polarities [24]. In this regard, the sign of the bulk DMI determines the polarity of the straightly transmitted skyrmion. This emphasizes again the importance of profound DMI studies, not limited to interfaces.

To avoid unsuitable sample designs, numerical estimations of the DMI need to be checked experimentally and further optimized, especially concerning its sign [25]. For thin film interfacial DMI systems, Brillouin light scattering is considered to be one of the most reliable techniques to determine DMI value and sign, since it measures directly the nonreciprocal spin-wave dispersion $[13,25]$. However, it is not suitable for bulk measurements because it only probes the surface. To determine the DMI in bulk systems like the noncentrosymmetric B20 compounds, polarized small-angle neutron scattering (SANS) or spherical neutron polarimetry (SNP) is commonly used [26-28]. By measuring the chiral term of the magnetic scattering intensity, polarized SANS and SNP give direct access to the handedness of the spiral structure and, thus, the DMI sign. However, these well-established measurement techniques for interfacial and bulk DMI are only applicable to spiral structures; thus, determining the sign of the DMI in weak ferromagnetic (WFM) materials with zero propagation vector is still to be solved.

The classical problem of weak ferromagnetism, microscopically driven by the same exchange coupling as the complex spiral structures discussed above, is a particularly attractive test case for developing new theoretical techniques, as the magnetic structures do not exhibit long magnetic periodicities [29]. These distinct boundary conditions resulting from a zero propagation vector clearly simplify theoretical calculations and numerical models without omitting or approximating the underlying exchange interactions. Also, experimental limitations, which might be imposed by the required high $q$ resolution for extremely long magnetic periodicities, do not occur. Furthermore, WFM materials are the classical DMI compounds in which this antisymmetric interaction was discovered. This draws a fundamental interest in the determination of their DMI sign.

The issue of determining the DMI sign in classical WFM materials has recently been addressed by Dmitrienko et al. [29]. They used magnetic resonant x-ray scattering at a synchrotron source to determine the sign of the DMI in iron borate $\left(\mathrm{FeBO}_{3}\right)$. For this novel experimental technique, the interference of two scattering processes is required: first, magnetic x-ray scattering with an external magnetic field applied in various directions to vary the alignment of the system's magnetic moment, and second, electric-quadrupole resonant scattering. Combining the results of both measurements, the authors [29] were able to relate the phase of the scattered waves from the magnetic and crystal structure and thus determine unambiguously the sign of the DMI in this compound. Although this advanced measurement technique has been successfully adopted to determine experimentally the DMI sign of other weak ferromagnets with $R \overline{3} c$ symmetry of type $M \mathrm{CO}_{3}$, where $M$ is a transition metal like, e.g., Mn, Co, Ni [30,31], it is rather complex and difficult to interpret.

A direct and more elegant approach to determine the phase relation between the magnetic and nuclear structure for compounds with zero propagation vector is offered by polarized neutron diffraction (PND). It gives direct access to the phase-sensitive interference term in the scattering cross section, which can be used to distinguish between a negative and positive sign of the DMI. Consequently, the experimental effort can be reduced to the measurement of a single flipping ratio (FR) [the intensity ratio for scattering with up (+) and down (-) polarized neutrons] on a mixed nuclear-magnetic Bragg reflection. In classical PND studies the main focus lies on determining the absolute value of the flipping asymmetry. The importance of its sign (which also depends on the used neutron polarization method) was generally neglected. In the present study, we show explicitly in a systematic and elegant way that this sign may be of crucial importance to reveal such fundamental information as the sign of the DMI. Although PND has already been utilized to determine the absolute direction of the magnetic moment in WFM materials [32-34], the number of investigated compounds is rather low and the DMI sign has not explicitly been determined. Moreover, there is so far no overlap with the compounds studied by magnetic resonant $\mathrm{x}$-ray scattering to allow a direct comparison of the two techniques.

Here, we report on a focused experimental study to determine for the first time with PND the sign of the DMI in two prototypical WFM compounds and provide results on 
other examples as well. The first prototypical compound, rhodochrosite $\left(\mathrm{MnCO}_{3}\right)$, which was previously measured by the mentioned complex resonant synchrotron technique [30], was selected to prove the consistency of the DMI sign obtained by the PND and x-ray methods. The second compound is the classical weak ferromagnet hematite $\left(\alpha-\mathrm{Fe}_{2} \mathrm{O}_{3}\right.$, sometimes called the mother of all antiferromagnets where the DMI has historically been first proposed $[1,2]$ ), for which the absolute sign of the DMI is disclosed for the first time. Interestingly, the occurrence of a new topological magnetic vortexlike structure called meron-antimeron pairs (half-skyrmions, carrying an outof-plane core magnetization) has been reported very recently in thin hematite films at room temperature [35]. Here, we provide the fundamental insight into one of the oldest known canted antiferromagnets, which may further improve the understanding for the occurrence of those novel topologic magnetic phenomena and their potential technological applicability. To demonstrate the generality of the presented PND method for a DMI sign determination in any WFM material with zero propagation vector, it is additionally applied to magnetocaloric, orthorhombic $\mathrm{HoFeO}_{3}$ and multiferroic, tetragonal, noncentrosymmetric $\mathrm{Ba}_{2} \mathrm{CoGe}_{2} \mathrm{O}_{7}$. The measured results are in good agreement with our detailed $a b$ initio density functional theory (DFT) calculations performed by using the QUANTUM ESPRESSO package for both title compounds.

\section{EXPERIMENTAL SETUP AND SAMPLES}

The PND experiments were carried out at the single crystal diffractometer POLI at the Heinz Maier-Leibnitz Zentrum (MLZ) in Garching [36,37]. Its newly developed FR setup option, consisting of a $2.2 \mathrm{~T}$ high- $T_{c}$ superconducting magnet, a ${ }^{3} \mathrm{He}$ polarizer, and a dedicated flipper, is described in detail in Refs. [38,39].

For the experiment, the asymmetry $A$ of all available mixed nuclear magnetic Bragg reflections were measured by determining the scattered intensity $I^{ \pm}$with the incoming neutron polarization either in the up $(+)$ or down $(-)$ state. The measured asymmetry is given by the ratio between the difference $\left(I^{+}-I^{-}\right)$and sum $\left(I^{+}+I^{-}\right)$of these intensities and is directly proportional to the nuclear-magnetic interference term of PND (note that the equivalent FR value is given by the direct ratio between $I^{+}$and $I^{-}$). As a result, the sign of the asymmetry contains information about the phase difference between the nuclear and magnetic structure, and thereby ultimately about the absolute sign of the DMI. A more detailed, theoretical representation of the measured asymmetry on the nuclear and magnetic structure factor is given in the Appendix.

The used high-quality single crystals of hematite with $5 \times 5 \times 4 \mathrm{~mm}^{3}$ size and an almost cubic shape, and of rhodochrosite, a $8 \times 12 \times 2 \mathrm{~mm}^{3}$ sized pallet with triangular shape, were well characterized in previous synchrotron investigations.

\section{SYMMETRY ANALYSIS}

The local crystal symmetry restricts the directions of the DMI and may even forbid a WFM moment under certain conditions. Therefore, a detailed symmetry analysis and a clear definition of the used atom configuration are a mandatory prerequisite before starting the experiment. Since the DMI sign is directly related to the phase difference between the nuclear and magnetic structure, it is possible to relate the absolute sign of the DMI to a specific magnetic moment arrangement, which could then be reliably determined by the PND experiment.

For both compounds studied in this work [detailed structure parameters and atom positions summarized in Supplemental Material (SM), Sec. I [40] ], the magnetic state can be described by two sublattices $A$ and $B$ with magnetic moments $\boldsymbol{m}_{A}$ and $\boldsymbol{m}_{B}$ and a zero propagation vector. The moments of each sublattice are antiparallel with respect to the other when the small canting, leading to a WFM moment of 0.0337(4) $\mu_{B} / \mathrm{Mn}$ for $\mathrm{MnCO}_{3}$ and around $0.00398(4) \mu_{B} / \mathrm{Fe}$ for $\alpha-\mathrm{Fe}_{2} \mathrm{O}_{3}$, is neglected [41-43]. The DMI energy between two such antiferromagnetic sublattices is usually given as their vector product multiplied with the vectorial DMI parameter $\boldsymbol{D}$ [3]. But using this definition, the sign of $\boldsymbol{D}$ cannot be uniquely defined, since it depends on our choice which sublattice is $A$ or $B$ [44]. To resolve this issue, the global interaction vector between the AFM sublattices is redefined on an atomic level by an antisymmetric $\boldsymbol{D}_{m n}$ for each magnetic moment pair $\boldsymbol{m}_{m}, \boldsymbol{m}_{n}$. This leads to a contribution of the DMI to the interaction energy of [30]

$$
\Delta E=\sum_{m \neq n} \boldsymbol{D}_{m n} \cdot\left(\boldsymbol{m}_{m} \times \boldsymbol{m}_{n}\right)
$$

Since the vector parameter $\boldsymbol{D}_{m n}$ is antisymmetric (i.e., satisfies $\boldsymbol{D}_{m n}=-\boldsymbol{D}_{n m}$ ), Eq. (1) is kept invariant under any spin interchange. As a result, Eq. (1) can be used to define the sign of the DMI. Similar to the original vector parameter $\boldsymbol{D}$, the entries of $\boldsymbol{D}_{m n}$ are restricted by the symmetry of the corresponding system [4]. Unfortunately, there is a lack in the literature on how to determine these restrictions on the DMI vector explicitly. Thus, a general approach for their determination in any crystal symmetry is discussed in Sec. III A and exemplarily carried out for both title compounds in the SM, Sec. II [40].

In general, the magnetizations $\boldsymbol{m}_{A}$ and $\boldsymbol{m}_{B}$ of the two sublattices can be split in a ferromagnetic (FM) part $\boldsymbol{m}_{A}^{\mathrm{FM}}=\boldsymbol{m}_{B}^{\mathrm{FM}}=\left(\boldsymbol{m}_{A}+\boldsymbol{m}_{B}\right) / 2$, pointing for both sublattices in the same direction, and an AFM part $\boldsymbol{m}_{A}^{\mathrm{AFM}}=-\boldsymbol{m}_{B}^{\mathrm{AFM}}=\left(\boldsymbol{m}_{A}-\boldsymbol{m}_{B}\right) / 2$, which reverses its sign between the sublattices (see detailed view in the dotted circles in Fig. 1). This simplifies the equation for the energy difference for an atom $m$ of sublattice $A$ due to DMI with atoms $n$ of sublattice $B$ to 

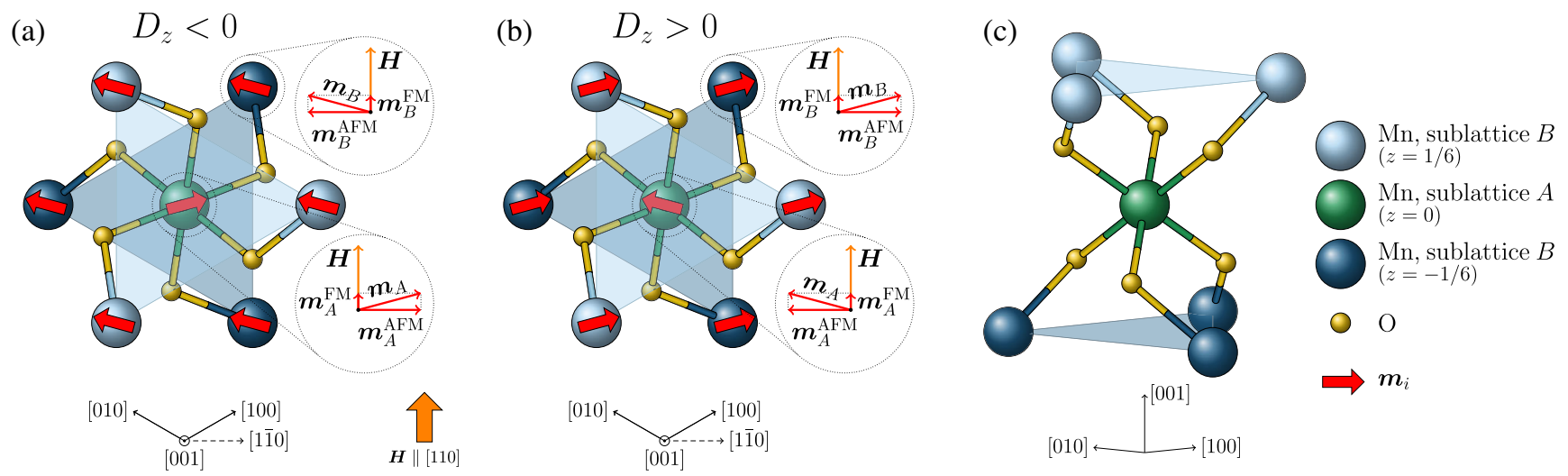

FIG. 1. The local environment of the manganese atom in the $z=0$ layer (green sphere) in the hexagonal unit cell of $\mathrm{MnCO}_{3}$. The six nearest-neighbor manganese atoms are shown as blue spheres, with the light blue atoms above $(z=1 / 6)$ and the dark blue atoms below $(z=-1 / 6)$ the central atom. The oxygen atoms $(z= \pm 1 / 12)$ between these manganese layers are shown as yellow spheres. The carbon atoms are omitted for clarity. Panels (a) and (b) show the two possible magnetic moment configurations which stabilize depending on the sign of the DMI by applying an external magnetic field $\boldsymbol{H}$ along the [110] direction and, thus, aligning the WFM moment along $\boldsymbol{H}$. The definition of $D_{z}$ is given in the text.

$$
\Delta E_{m}=-2 \sum_{n} \boldsymbol{D}_{m n} \cdot\left(\boldsymbol{m}_{A}^{\mathrm{FM}} \times \boldsymbol{m}_{A}^{\mathrm{AFM}}\right)
$$

Therefore, the DMI sign directly couples the alignment of the AFM structure to $\boldsymbol{m}_{A}^{\mathrm{FM}}$, which consists of the WFM moment and a magnetic moment eventually induced by an external field. In order to minimize the system's energy, the components of $\boldsymbol{D}_{m n}$ must have the same sign as $\boldsymbol{m}_{A}^{\mathrm{FM}} \times \boldsymbol{m}_{A}^{\mathrm{AFM}}$.

Moreover, due to the higher symmetry of the magnetic lattice compared to the symmetry of the complete unit cell in both studied compounds, $\boldsymbol{m}_{A}^{\mathrm{FM}}$ contributes only to $(h k l)$ Bragg reflections with even $l$ indices (so-called FM-type reflections), whereas $\boldsymbol{m}_{A}^{\mathrm{AFM}}$ contributes only to the reflections with odd $l$ indices (AFM-type reflections). Thus, each part of the magnetization can be independently investigated by diffraction.

\section{A. Symmetry restrictions on the DMI vector}

The DMI may be expected to transform as an axial vector. This means that if two sites $\boldsymbol{r}_{1}$ and $\boldsymbol{r}_{2}$ are connected by the DMI vector $\boldsymbol{D}_{12}$, then the sites $\boldsymbol{r}_{S 1}$ and $\boldsymbol{r}_{S 2}$, resulting from $\boldsymbol{r}_{1}$ and $\boldsymbol{r}_{2}$ by symmetry operation $\mathcal{S}$, are connected by

$$
\boldsymbol{D}_{S 1 S 2}=\operatorname{det}[\mathcal{R}] \mathcal{R}\left[\boldsymbol{D}_{12}\right],
$$

where $\mathcal{R}$ is the point group symmetry part of $\mathcal{S}$. This does not only relate the DMI vectors of symmetry-equivalent site pairs, but also imposes symmetry restrictions on $\boldsymbol{D}_{12}$, if $\mathcal{S}$ interchanges $\boldsymbol{r}_{1}$ and $\boldsymbol{r}_{2}$ or leaves them unchanged. For an interchange of the two sites, e.g., by an inversion center between them, Eq. (3) can be reduced to $\boldsymbol{D}_{12}=-\operatorname{det}[\mathcal{R}] \mathcal{R}\left[\boldsymbol{D}_{12}\right]$, utilizing the antisymmetric properties of $\boldsymbol{D}$. If the sites are unchanged by $\mathcal{S}$, e.g., as for a mirror plane including $\boldsymbol{r}_{1}$ and $\boldsymbol{r}_{2}$, Eq. (3) reduces to $\boldsymbol{D}_{12}=\operatorname{det}[\mathcal{R}] \mathcal{R}\left[\boldsymbol{D}_{12}\right]$. Using these two relations, we can obtain all the symmetry restrictions on $\boldsymbol{D}$ found by Moriya [4] as demonstrated in the SM, Sec. II [40]. This proves that $\boldsymbol{D}$ is an axial vector.

\section{B. Crystal structure and DMI in rhodochrosite $\left(\mathrm{MnCO}_{3}\right)$}

The $R \overline{3} c$ crystal structure $(a \approx 4.8 \AA$ and $c \approx 15.6 \AA$ in the hexagonal setting, detailed in SM, Sec. I A [40]) of $\mathrm{MnCO}_{3}$ is sketched in Fig. 1, with the manganese atoms belonging to sublattices $A$ and $B$ colored green and blue, respectively. The stacking of the manganese atoms is such that there would be a center of inversion between neighboring manganese atoms, and according to the symmetry implications provided by Moriya [4], no DMI possible. However, the oxygen atoms (colored yellow in Fig. 1) clearly break these local inversion centers, allowing a finite DMI vector between the magnetic manganese atoms of neighboring layers (green and light blue layers at $z=0$ and $z=1 / 6$ in Fig. 1). Because of the threefold inversion axis of the $R \overline{3} c$ crystal symmetry, the in-plane components of the DMI vectors cancel out in the sum of Eq. (2), as discussed in detail in the SM, Sec. II B [40]. Since the remaining nonzero $z$ components are equal for the DMI vectors between the $z=0$ and the $z= \pm 1 / 6$ layers, the individual DMI vectors can finally be replaced by a symmetry averaged $\overline{\boldsymbol{D}}_{0 \frac{1}{6}}$, which is parallel to the $z$ axis. To adopt the sign convention for $\boldsymbol{D}$ used by Beutier et al. [30] and Dmitrienko et al. [29], we define $\boldsymbol{D}=\overline{\boldsymbol{D}}_{0 \frac{1}{6}}$ with only $D_{z}$ nonzero. Using Eq. (2), this results in an energy change of $\Delta E=-12 D_{z}\left(\boldsymbol{m}_{A}^{\mathrm{FM}} \times \boldsymbol{m}_{A}^{\mathrm{AFM}}\right)_{z}$ per manganese atom due to the DMI with its six nearest neighbors. 

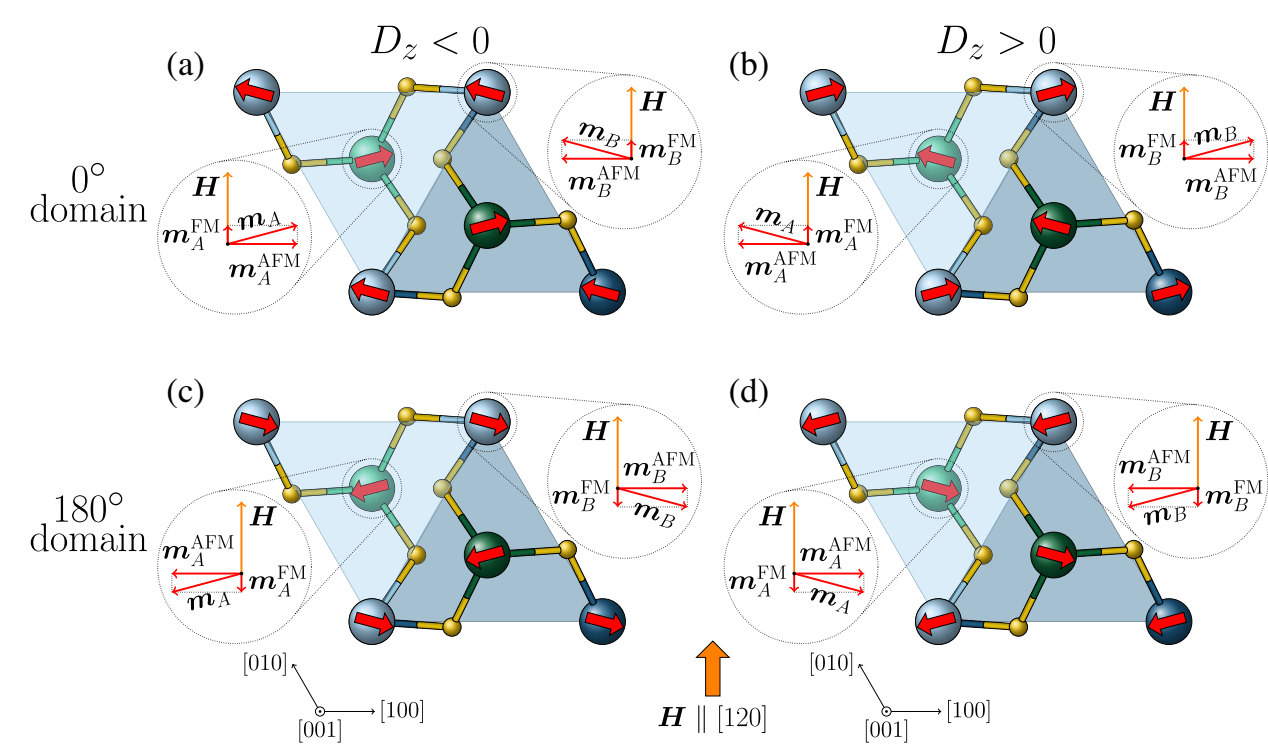

(d)

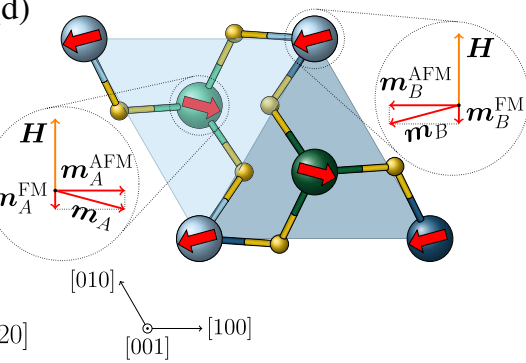

(e)

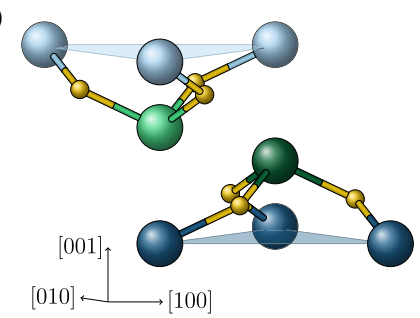

FIG. 2. The local environment of the iron atoms of the $z=0$ layer (light and dark green spheres) in the hexagonal unit cell of $\alpha$-Fe ${ }_{2} \mathrm{O}_{3}$. The three nearest-neighbor iron atoms of the $z= \pm 1 / 6$ layer are shown as blue spheres, with the light blue atoms above $\left(z=+1 / 6-\Delta z_{\mathrm{Fe}}\right)$ and the dark blue atoms below $\left(z=-1 / 6+\Delta z_{\mathrm{Fe}}\right)$ the central layer. The oxygen atoms $(z= \pm 1 / 12)$ between these iron layers are shown as yellow spheres. Panels (a)-(d) show the two possible magnetic moment configurations (a),(b) and their $180^{\circ}$ domains (c),(d), which could stabilize depending on the sign of the DMI by applying an external magnetic field $\boldsymbol{H}$ along the [120] direction. By aligning the WFM moment along $\boldsymbol{H}$, configurations (a) and (b) are energetically favored to their $180^{\circ}$ domains. The definition of $D_{z}$ is given in the text.

Since the spins of the AFM structure below $T_{N} \approx 32.4 \mathrm{~K}$ are confined to the basal plane, perpendicular to the threefold axis $[43,45]$, the nonzero $D_{z}$ component causes an in-plane tilt of the spins. To be energetically favored, $D_{z}$ must have the same sign as $\left(\boldsymbol{m}_{A}^{\mathrm{FM}} \times \boldsymbol{m}_{A}^{\mathrm{AFM}}\right)_{z}$. By applying an external magnetic field in the $a b$ plane larger than $0.2 \mathrm{~T}$, a single domain state is obtained with WFM moment aligned along the field direction [43,46]. Thus, $\boldsymbol{m}_{A}^{\mathrm{FM}}$ is oriented parallel to the applied magnetic field and a particular $\boldsymbol{m}_{A}^{\mathrm{AFM}}$ direction is adopted for $D_{z}<0$ and $D_{z}>0$, respectively, in order to fulfill $D_{z}\left(\boldsymbol{m}_{A}^{\mathrm{FM}} \times \boldsymbol{m}_{A}^{\mathrm{AFM}}\right)_{z}>0$.

For a magnetic field along the crystal [110] direction, as it was the case for the performed PND experiment presented in Sec. IVA, this results in the two possible magnetic moment configurations shown in Figs. 1(a) and 1(b). Note that the orientation of the applied field in the $a b$ plane does not need to represent the direction of the WFM moment in the ground state, which is discussed in the SM, Sec. VI [40]. Any field alignment resulting in a welldefined, quasimonodomain state of the magnetic structure reflects the sign of the DMI, which is the same without and with an applied field, correctly.

\section{Crystal structure and DMI in hematite $\left(\alpha-\mathrm{Fe}_{2} \mathrm{O}_{3}\right)$}

The $R \overline{3} c$ crystal structure of $\alpha-\mathrm{Fe}_{2} \mathrm{O}_{3}(a \approx 5.0 \AA$ and $c \approx$ $13.8 \AA$ in the hexagonal setting, detailed in SM, Sec. IB [40]) is sketched in Fig. 2 and similarly layered as in
$\mathrm{MnCO}_{3}$. The iron atoms in $\alpha-\mathrm{Fe}_{2} \mathrm{O}_{2}$ are also arranged in layers of alternating $A$ and $B$ sublattice type (green and blue in Fig. 2), but each of the layers is occupied with two iron atoms, one shifted slightly above and the other below (light and dark green spheres in Fig. 2, respectively). Because of this slight shift, every iron atom has only three nearest neighbors from the next layer above or below (light and dark blue spheres in Fig. 2). Again, there would be an inversion center between neighboring iron atoms from different layers, and thus no DMI possible, but these are clearly broken by the oxygen atoms (yellow spheres in Fig. 2). As already observed for $\mathrm{MnCO}_{3}$, the threefold symmetry imposed by space group $R \overline{3} c$ leads to a cancellation of the in-plane components of the DMI vector in the sum of Eq. (2). Thus, the individual DMI vectors can be replaced by a symmetry averaged $\overline{\boldsymbol{D}}_{0 \frac{1}{6}}$, which is parallel to the $z$ axis, as discussed in detail in SM, Sec. II C [40]. This reduces the DMI energy per magnetic layer containing two iron atoms in Eq. (2) to $\Delta E=-12 D_{z}\left(\boldsymbol{m}_{A}^{\mathrm{FM}} \times \boldsymbol{m}_{A}^{\mathrm{AFM}}\right)_{z}$, using the same convention $\boldsymbol{D}=\overline{\boldsymbol{D}}_{0 \frac{1}{6}}$ as for $\mathrm{MnCO}_{3}$ in the preceding section.

The basic AFM structure of $\alpha-\mathrm{Fe}_{2} \mathrm{O}_{3}$ has been solved by Shull et al. [47] with neutron powder diffraction. Above the Morin temperature $T_{M} \approx 259.1(2) \mathrm{K}$ up to $T_{N} \approx 955 \mathrm{~K}$, the spins are confined to the hexagonal basal plane forming a WFM structure similar to $\mathrm{MnCO}_{3}$ [48]. However, the WFM moment in $\alpha-\mathrm{Fe}_{2} \mathrm{O}_{3}$ is over 8 times smaller than in $\mathrm{MnCO}_{3}$ and therefore more difficult to measure. Below $T_{M}$, the magnetic structure undergoes a spin flop transition and 
the weak ferromagnetism is absent. As we are interested in the DMI sign, we focus on the room-temperature WFM phase, stabilized between $T_{M}$ and $T_{N}$.

In zero magnetic field, $\alpha-\mathrm{Fe}_{2} \mathrm{O}_{3}$ has $180^{\circ}$ domains within each of the three trigonal domains, thus in total six equally populated AFM domains [49,50]. By applying an external magnetic field in the hexagonal $a b$ plane, the AFM moments align perpendicular to the field direction and the trigonal domains vanish. This was confirmed by uniaxial neutron polarization analysis (PA) on the purely magnetic (003) Bragg reflection [38,49]. Considering the remaining two $180^{\circ}$ domains, which cannot be resolved by a PA experiment on a purely magnetic reflection, and either a positive or negative sign for $D_{z}$, there are in total four possible spin arrangements. For a magnetic field along the crystal [120] direction, as it was the case for the performed PND experiment presented in Sec. IV B, these four possible magnetic moment configurations are shown in Figs. 2(a)-2(d). Because of a parallel alignment of the WFM moment with the field direction, configurations of Fig. 2(a) or 2(b) are energetically more favored, dependent on a negative or positive sign of $D_{z}$, respectively. However, this energy difference to their $180^{\circ}$ domain is considerably lower than in $\mathrm{MnCO}_{3}$ due to the comparatively small WFM moment in $\alpha-\mathrm{Fe}_{2} \mathrm{O}_{3}$.

\section{EXPERIMENTAL RESULTS}

\section{A. DMI sign and WFM structure of $\mathrm{MnCO}_{3}$ - Method validation}

PND measurements on a $\mathrm{MnCO}_{3}$ single crystal were performed at $4 \mathrm{~K}$ in the WFM state. The crystal was mounted such that the [110] direction is normal to the horizontal plane, and thus parallel to the applied magnetic field of $2.2 \mathrm{~T}$. This gives access to in-plane Bragg reflections of $(h \bar{h} l)$ type and aligns $\boldsymbol{m}_{A}^{\mathrm{FM}}$ along [110]. The AFM spin structure is expected to be in the $a b$ plane perpendicular to the field direction, thus parallel or antiparallel to the [110] direction, dependent on the sign of the DMI, as illustrated in Figs. 1(a) and 1(b). Because of the used high magnetic field value of $2.2 \mathrm{~T}$, which is clearly above the $0.2 \mathrm{~T}$ required for a single domain state, $180^{\circ}$ domains, as shown for $\alpha-\mathrm{Fe}_{2} \mathrm{O}_{3}$ in Figs. 2(c) and 2(d), are not expected [43].

To relate the sign of the experimentally measured asymmetry to a certain spin configuration of Fig. 1, and thus to the sign of the DMI, the nuclear and magnetic structure factors given in the Appendix need to be evaluated. In a previous PND study on $\mathrm{MnCO}_{3}$ by Brown and Forsyth [46] only the asymmetries of FM-type reflections are reported. Thus, these results only give information about $\boldsymbol{m}_{A}^{\mathrm{FM}}$, which is not sufficient to extract the sign of the DMI. In the current experiment, we measured the asymmetry of 298 reflections, 104 of AFM and 194 of FM type. We take the strong, out-of-plane AFM-type Bragg reflection $(3 \overline{2} \overline{1})$ as an example for our calculations (note that for in-plane AFM-type Bragg reflections $\boldsymbol{P} \boldsymbol{M}_{\perp \boldsymbol{q}}$ is always zero; thus no asymmetry measurable). Using Eqs. (A3)-(A5) from the Appendix, we can relate the AFM moment configuration from Figs. 1(a) and 1(b) with a positive and negative sign of $A_{3 \overline{1}}$, respectively. This is explicitly demonstrated in the SM, Sec. III A [40]. Since the measured asymmetry with a value of $0.237(3)$ is clearly positive, we can determine as the adopted spin configuration the one shown in Fig. 1(a) and therefore deduce a negative sign of the DMI $\left(D_{z}<0\right)$. This is in agreement with the magnetic resonant $\mathrm{x}$-ray scattering results by Beutier et al. [30]. Thus, the measured polarized neutron asymmetry in applied magnetic field on a single suitable Bragg reflection already provides the answer to the fundamental question about the absolute direction of the DMI in this compound. Since the measurement time for the high precision asymmetry obtained for the $(3 \overline{2} \overline{1})$ Bragg reflection was below 2 minutes, such a measurement is easy to perform within a short time, demonstrating the power and convenience of the PND method.

In order to show that not only the $(3 \overline{2} \overline{1})$ reflection supports the negative sign of the $\mathrm{DMI}$ in $\mathrm{MnCO}_{3}$ and in fact any sufficiently strong out-of-plane AFM-type reflection can be used for the DMI sign determination, we precisely refined $\boldsymbol{m}_{A}^{\mathrm{FM}}$ and $\boldsymbol{m}_{A}^{\mathrm{AFM}}$ from the complete set of measured asymmetry values. For this refinement, the asymmetry values of the 54 Bragg reflections with the lowest intensity (below $1.0 \%$ of the maximal observed intensity) were excluded due to their high measurement uncertainty. Of the remaining 244 Bragg reflections, 147 were of FM type and 97 of AFM type. Although the vertical axis of the instrument, and thus the field and polarization axis, was aligned rather well with the crystal [110] direction, a small misalignment of $1.7(3)^{\circ}$ within the $a b$ plane toward the $b$ axis (Fig. 3) and $1.2(5)^{\circ}$ toward the $c$ axis was observed by refining the experimental orientation matrix and considered for the refinement. The refinement was performed using the Mag2Pol program [51] and validated with the JANA program [52] and the CHILSQ subroutine of the Cambridge Crystallographic Subroutine Library (CCSL) [53]. A major advantage of the refinement on asymmetries rather than intensities is the invariance of the collected asymmetries to the absolute intensity of the individual Bragg reflections. Thus, the influence of parasitic effects like absorption (crystal shape), multiple scattering, etc., are minimized. Nevertheless, extinction was taken into account for all refinements.

Starting with the results of the restriction-free refinement listed in Table I, we observe for $\boldsymbol{m}_{A}^{\mathrm{FM}}$ only a significant contribution in $x$ direction, which is defined to be parallel to the applied field direction (see Fig. 3). This agrees well with our expectations, since the FM moment is composed of the intrinsic aligned WFM moment and the external field induced moment, both parallel to the field. Additionally, 

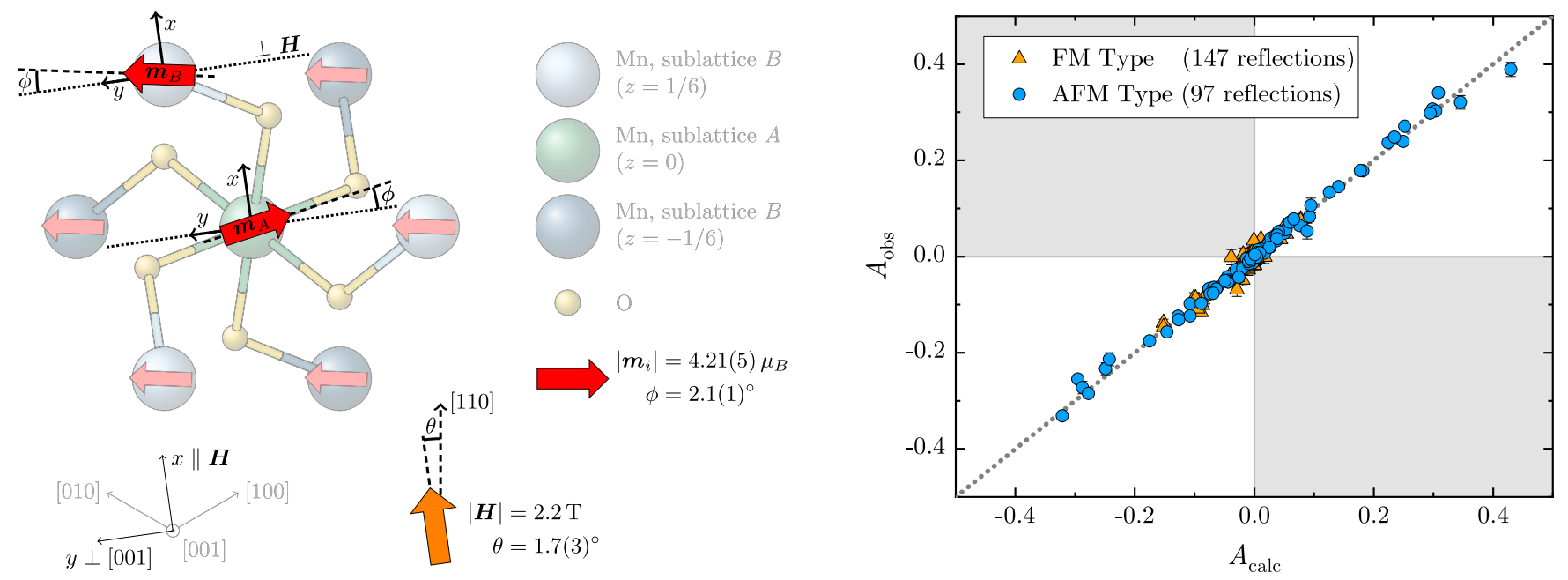

FIG. 3. Visualized results of the symmetry restricted magnetic moment refinement in $\mathrm{MnCO}_{3}$ from Table I. The left-hand side shows the projection of the exact field direction (orange arrow) and the resulting magnetic moment configuration (red arrows) on the $a b$ plane. The magnetic field has a small out-of-plane component of $1.2(5)^{\circ}$ toward the $c$ axis. All angles are drawn exaggerated to be visible. Additionally, the orthogonal $x, y$, and $z$ directions used in the text are defined. The right-hand side shows the comparison between the observed and calculated asymmetry values for the refinement.

this FM component offers a convenient and fast crosscheck for the assumed field and polarization directions. If one of them is assumed by mistake in the opposite direction, which could lead to a wrong determination of the sign of the DMI, we would observe a negative FM contribution along $x$. But this is clearly not the case. For $\boldsymbol{m}_{A}^{\mathrm{AFM}}$, the highest contribution is found along the $y$ direction, which is perpendicular to the magnetic field and within the $a b$ plane. A rather small magnetization is observed along the $z$ direction, which is the right-handed completion of $x$ and $y$ and approximately parallel to the crystal [001] direction, leading to a small tilt of $1.7(5)^{\circ}$ for the AFM moment out of the $a b$ plane. This is in agreement with the theoretical expectations by Dzyaloshinskii [1], predicting a slight out-of-plane tilt up to the order of $1^{\circ}$ for a $\mathrm{C} 2 / \mathrm{c}$ ground state symmetry, which is discussed in more detail in the SM, Sec. VI [40]. There is no significant AFM moment observed along $x$, the field direction. The magnitude of the AFM moment in $\mathrm{MnCO}_{3}$ of $4.23(6) \mu_{B}$, which was experimentally determined for the first time, agrees

TABLE I. Overview of the results of the refinement using free or symmetry restricted magnetic moments for the reduced dataset with 244 measured asymmetries in $\mathrm{MnCO}_{3}$ at $4 \mathrm{~K}$. The components of the magnetization refer to the $x y z$ directions defined in Fig. 3.

\begin{tabular}{lclccc}
\hline \hline Component & Restriction & $m_{x}\left(\mu_{B}\right)$ & $m_{y}\left(\mu_{B}\right)$ & $m_{z}\left(\mu_{B}\right)$ & $\chi_{r}^{2}$ \\
\hline $\boldsymbol{m}_{A}^{\mathrm{FM}}$ & Free & $0.16(1)$ & $-0.02(6)$ & $0.02(4)$ & 6.00 \\
$\boldsymbol{m}_{A}^{\mathrm{AFM}}$ & Free & $0.02(1)$ & $-4.23(6)$ & $0.12(4)$ & 4.69 \\
$\boldsymbol{m}_{A}^{\mathrm{FM}}$ & $\| \boldsymbol{H}$ & $0.156(8)$ & 0 & 0 & 5.98 \\
$\boldsymbol{m}_{A}^{\mathrm{AFM}}$ & $\perp \boldsymbol{H}$ & 0 & $-4.21(5)$ & $0.13(4)$ & 4.70 \\
\hline \hline
\end{tabular}

well with the expectations for the ordered moment of $\mathrm{Mn}^{2+}$ observed in other compounds (e.g., $4.05 \mu_{B}$ in $\mathrm{MnGeO}_{3}$ or $4.55 \mu_{B}$ in $\mathrm{MnTiO}_{3}$ [54,55]). This confirms that no $180^{\circ}$ domains are present, since otherwise the magnetic moment would be clearly reduced, as we will see for $\alpha-\mathrm{Fe}_{2} \mathrm{O}_{3}$ in the next section. Since the FM and AFM moments contribute to different types of reflections, the reduced chi squared value $\chi_{r}^{2}$ is given for each group of reflections separately. Considering these results from the free refinement, it is reasonable to restrict $\boldsymbol{m}_{A}^{\mathrm{FM}}$ parallel to the field direction and $\boldsymbol{m}_{A}^{\mathrm{AFM}}$ perpendicular to it. As shown in Table I, these restrictions result in no significant change of both refined moments and fit quality.

A visualization of the results from the restricted refinement, projected to the $a b$ plane, is shown in the left-hand panel of Fig. 3. By comparing these results with the two possible spin configurations for a plus and minus sign of the DMI, illustrated in Figs. 1(a) and 1(b), we can clearly confirm the negative sign for the DMI in $\mathrm{MnCO}_{3}$ already obtained from the inspection of the single $(3 \overline{2} \overline{1})$ Bragg reflection. Furthermore, the right-hand panel of Fig. 3 shows the very good agreement between the observed and calculated asymmetry values for the symmetry restricted refinement. It is important to note that all the asymmetry points of the AFM-type reflections within the two white areas support the refined sign of the DMI, whereas asymmetry points in the gray areas would support an inverted sign. Similarly, FM-type reflections in the white or gray areas support a parallel or antiparallel alignment of $\boldsymbol{m}_{A}^{\mathrm{FM}}$ to the field, respectively. This reemphasizes that it is in general sufficient to measure one strong AFM-type reflection with a significant asymmetry to reliably answer the question about the absolute direction of the DMI. This is 

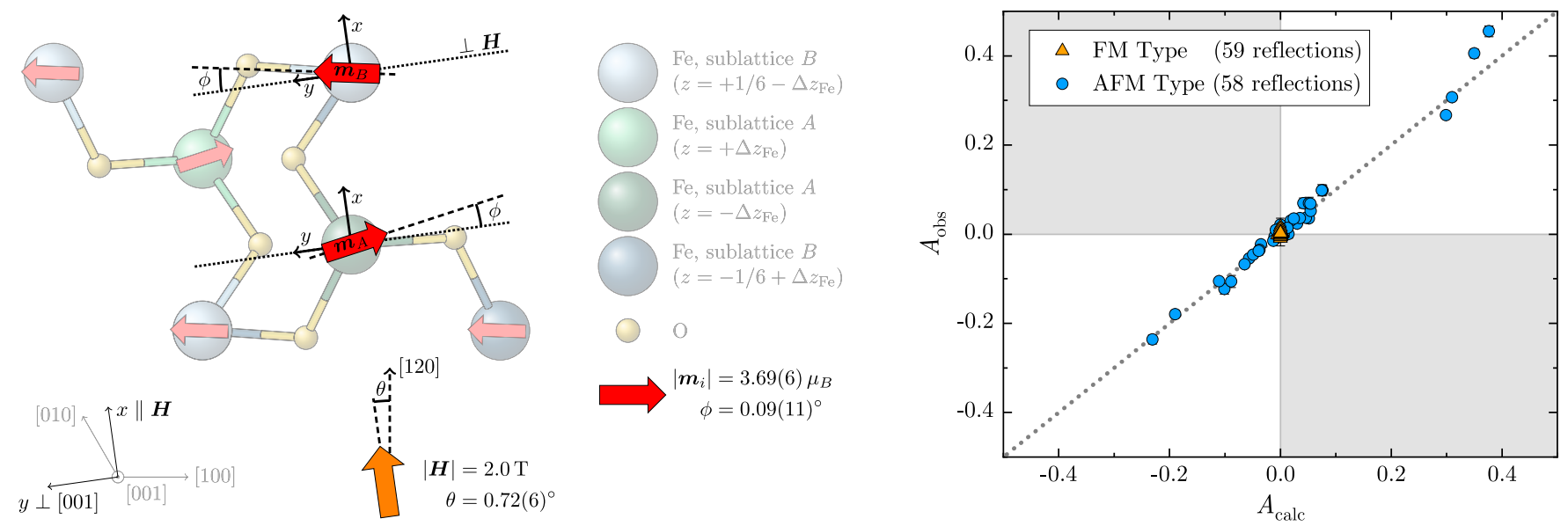

FIG. 4. Visualized results of the symmetry restricted magnetic moment refinement in $\alpha-\mathrm{Fe}_{2} \mathrm{O}_{3}$ from Table II. The left-hand side shows the projection of the exact field direction (orange arrow) and the resulting magnetic moment configuration (red arrows) on the $a b$ plane. The magnetic field has a small out-of-plane component of $-0.7(1)^{\circ}$ toward the $c$ axis. All angles are drawn exaggerated to be visible. Additionally, the orthogonal $x, y$, and $z$ directions used in the text are defined. The right-hand side shows the comparison between the observed and calculated asymmetry values for the refinement.

especially important for compounds, for which the accessible $q$ range is too small or the counting rates are too low for the collection of enough data for a complete refinement.

\section{B. DMI sign and WFM structure of $\alpha-\mathrm{Fe}_{2} \mathrm{O}_{3}$}

Because hematite is the textbook example of a canted AFM material, it has been studied exhaustively over many years. However, early PND studies by Nathans et al. [49] revealed a puzzling behavior for Friedel pairs in this compound: The authors observed nonequivalent PND asymmetry values for reflections belonging to the same Friedel pair, some even with an opposite sign, which is not allowed by the centrosymmetric $R \overline{3} c$ crystal symmetry in $\alpha-\mathrm{Fe}_{2} \mathrm{O}_{3}$. Because of this controversial result, it was so far not possible to extract the sought sign of the DMI.

To investigate this issue, we performed a systematic study of the AFM-type Bragg reflection (2113) [which is (210) in the rhombohedral unit cell used by Nathans et al. [49] ] and its symmetry equivalents. We measured the asymmetry values of four symmetry-equivalent (21̄3)-type Bragg reflections for different crystal alignments with the vertical axis of the instrument close to the [120] direction. Overall, we could reproduce the previous results and observed an almost constant offset between the measured asymmetries within Friedel pairs, which leads to a sign change for a good vertical alignment of the [120] direction. This offset can be perfectly understood by a small misalignment of the magnetic field direction to the vertical sample rotation axis of the instrument. Correcting for this measurement artifact, we observe equal asymmetries within Friedel pairs as expected by the crystal symmetry. Thus, explaining the discrepancies observed in Ref. [49] preventing so far the determination of the DMI sign, we are finally able to unambiguously extract the DMI sign in $\alpha-\mathrm{Fe}_{2} \mathrm{O}_{3}$ as already done for $\mathrm{MnCO}_{3}$ in the previous section. More details about this study can be found in the SM, Sec. IV [40].

For $\alpha-\mathrm{Fe}_{2} \mathrm{O}_{3}$, we collected the asymmetry value of 126 Bragg reflections (64 AFM and 62 FM) in the WFM state at room temperature. The crystal [120] direction was aligned vertically, thus parallel to the applied magnetic field of $2.0 \mathrm{~T}$. This gives access to Bragg reflections of $(2 h \bar{h} l)$ type in the horizontal scattering plane. Because of the preferred alignment of the WFM moment along the field direction, either of the spin configurations illustrated in Figs. 2(a) and 2(b) should be present, dependent on the noted sign of the DMI in $\alpha-\mathrm{Fe}_{2} \mathrm{O}_{3}$. In order to decide between these two configurations, we investigated the strong out-of-plane

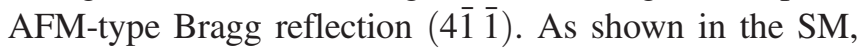
Sec. IV [40], the influence of the mentioned small misalignment of the magnetic field is negligible for this reflection. Using Eqs. (A3)-(A5) from the Appendix, we can relate the AFM moment configuration from Figs. 2(a) and 2(b) with a positive and negative sign of $A_{4 \overline{1} \overline{1}}$, respectively. This is explicitly demonstrated in the SM, Sec. III B [40]. Since the measurement revealed a clearly positive asymmetry value of 0.41(1), we can determine as the adopted configuration the one shown in Fig. 2(a) and doubtless conclude about a negative sign for the DMI in $\alpha-\mathrm{Fe}_{2} \mathrm{O}_{3}$.

For a further verification of our results and to reemphasize that any AFM-type Bragg reflection with sufficiently strong asymmetry is suitable for the DMI sign determination by PND, we refined the magnetic moment values and directions from the complete set of measured asymmetry values as done in Sec. IVA for $\mathrm{MnCO}_{3}$. For the refinement with the Mag2Pol program [51], the nine lowestintensity reflections (below $1.7 \%$ of the maximal observed 
TABLE II. The results for the magnetic moment refinement for the predominant domain in $\alpha-\mathrm{Fe}_{2} \mathrm{O}_{3}$ from the reduced dataset of 117 measured PND asymmetries both in the free and restricted models. The minority domain, accounting for a fraction of $17(2) \%$ for the free and $16.0(7) \%$ for the restricted refinement, has a spin structure rotated by $180^{\circ}$ around the $c$ axis. The components of the magnetization refer to the $x y z$ directions defined in Fig. 4. A corresponding refinement in a single domain state can be found in the SM, Sec. V [40], which shows a significantly lower fit quality and a clearly reduced total magnetic moment.

\begin{tabular}{lclccc}
\hline \hline Component & Restriction & $m_{x}\left(\mu_{B}\right)$ & $m_{y}\left(\mu_{B}\right)$ & $m_{z}\left(\mu_{B}\right)$ & $\chi_{r}^{2}$ \\
\hline $\boldsymbol{m}_{A}^{\mathrm{FM}}$ & Free & $0.01(1)$ & $0.0(2)$ & $0.00(5)$ & 0.78 \\
$\boldsymbol{m}_{A}^{\mathrm{AFM}}$ & Free & $0.03(1)$ & $-3.9(2)$ & $-0.17(5)$ & 2.44 \\
$\boldsymbol{m}_{A}^{\mathrm{FM}}$ & $\| \boldsymbol{H}$ & $0.006(7)$ & 0 & 0 & 0.79 \\
$\boldsymbol{m}_{A}^{\mathrm{AFM}}$ & $\perp \boldsymbol{H}$ & 0 & $-3.69(6)$ & $-0.18(3)$ & 2.79 \\
\hline \hline
\end{tabular}

intensity) were excluded due to their high measurement uncertainty, leaving $59 \mathrm{FM}$ - and 58 AFM-type reflections. Although the crystal [120] direction was aligned rather well with the vertical instrument axis, a slight tilt of $0.72(6)^{\circ}$ in the $a b$ plane toward the $b$ axis (Fig. 4) and $-0.7(1)^{\circ}$ toward the $c$ axis was observed by refining the experimental orientation matrix. This was taken into account for the refinement. Similar as for $\mathrm{MnCO}_{3}$, we refined the collected asymmetry values using either free or restricted $\left(\boldsymbol{m}_{A}^{\mathrm{FM}} \| \boldsymbol{H}\right.$ and $\left.\boldsymbol{m}_{A}^{\mathrm{AFM}} \perp \boldsymbol{H}\right)$ magnetic moments. Assuming a single domain state, the AFM moment of the free refinement was found to be only $2.37(6) \mu_{B}$ per iron atom (see SM, Sec. V, for detailed refinement results [40]). This is significantly lower than expected because, for example, neutron powder diffraction found an ordered AFM moment of 4.06(1) $\mu_{B}$ [56]. Because trigonal domains can be excluded (see Sec. III C), some of the $180^{\circ}$ domains must remain even after applying a magnetic field of $2.0 \mathrm{~T}$ to explain the observed discrepancies in the AFM moment. Introducing these $180^{\circ}$ domains in the refinement, which are illustrated in Fig. 2(c) or 2(d) dependent on a negative or positive DMI sign, respectively, increased drastically the fit quality for the AFM part from $\chi_{r}^{2}=3.63$ without to $\chi_{r}^{2}=2.44$ with domains. Moreover, the resulting AFM moments with 3.9(2) $\mu_{B}$ for the free and 3.69(6) $\mu_{B}$ for the restricted refinement (see Table II) are in clearly better agreement with literature when considering domains. The fraction of the $180^{\circ}$ domain was refined to be $17(2) \%$ for the free and 16.0(7)\% for the restricted refinement. Assuming an equal population of all six domains in zero field (see Sec. IIIC), the observed 1/6 domain ratio suggests that the initial domain with the WFM moment antiparallel to the field direction and the antiferromagnetism perpendicular to it still survives the application of the $2 \mathrm{~T}$ magnetic field, resulting in a quasimonodomain structure with $5 / 6$ of the sample volume in a certain AFM domain state and $1 / 6$ of the sample volume in the equivalent $180^{\circ} \mathrm{AFM}$ domain. This might be attributed to the smallness of the WFM moment in $\alpha-\mathrm{Fe}_{2} \mathrm{O}_{3}$ at RT compared to the $\mathrm{MnCO}_{3}$ experiment at $4 \mathrm{~K}$, finally not being sufficient to introduce a $180^{\circ}$ rotation of this domain. The detailed magnetic moment components refined for the predominant domain are listed in Table II.

Both refinements result in a small FM moment at the level of its uncertainty. This is not surprising as the expected FM component is of around $0.009 \mu_{B} / \mathrm{Fe}$ only, as determined from SQUID measurements in $2 \mathrm{~T}$ [41]. Such magnetic moment values indicate the sensitivity limit for the relatively short measurement times (on average $80 \mathrm{~s}$ for each Bragg reflection) used in this experiment. Among the measured $59 \mathrm{FM}$ asymmetries, only four show an asymmetry value larger than $2 \sigma$, but still below the $3 \sigma$ level. This explains also the small $\chi_{r}^{2}$ values for the FM part in Table II. However, since the FM moment direction is given by the field direction, a fact confirmed also from the $\mathrm{MnCO}_{3}$ refinement above, only a refinement of the AFM moment direction is crucial to verify the sign of the DMI.

For the AFM moment of the free refinement, we observe a small component along the $x$ direction, which might be attributed to the nonperfect field alignment. For the symmetry restricted refinement, this component is not allowed, leading to a slight increase in the $\chi_{r}^{2}$ value of the AFM part. For the $y$ and $z$ component, both refinements agree within the uncertainty. Again, the small $z$ component of the refined $\boldsymbol{m}_{A}^{\mathrm{AFM}}$ causes a slight tilt of the AFM moment by $2.8(5)^{\circ}$ out of the $a b$ plane, which is in accordance with the theoretical considerations by Dzyaloshinskii [1] and discussed in more detail in the SM, Sec. VI [40]. The results of the restricted refinement, projected to the $a b$ plane, are shown in Fig. 4. By comparing these results with the two possible spin configurations for a minus and plus sign of the DMI and an aligned WFM moment, illustrated in Figs. 2(a) and 2(b), respectively, one can clearly deduce a negative sign for the DMI in hematite. Moreover, the comparison between the observed and calculated asymmetry values in the right-hand panel of Fig. 4 shows a very good agreement for the AFM-type reflections, whereas almost no significant asymmetry for the FM-type reflections is observed. Note that all AFM-type asymmetry values within the two white areas support the refined sign of the DMI, whereas the ones in the gray areas would support an inverted sign. Therefore, we can select any AFM-type reflection with a significantly strong asymmetry to conclusively determine the negative sign of the DMI in $\alpha-\mathrm{Fe}_{2} \mathrm{O}_{3}$, emphasizing again the power of the PND method, even for the case where the WFM moment is extremely difficult to detect.

\section{DMI AND MAGNETIZATION VECTOR SIMULATION IN $\mathrm{MnCO}_{3}$ AND $\alpha$-Fe $\mathrm{F}_{2} \mathrm{O}_{3}$ BY AB INITIO CALCULATIONS}

In the case of $\mathrm{MnCO}_{3}$, the negative DMI sign determined by PND in Sec. IV A can be independently verified by the 
TABLE III. Search for a magnetic anisotropy in $\mathrm{MnCO}_{3}$. Magnetization vectors for different in-plane orientations of the WFM moment. The $x$ axis is along the crystal [100] direction and the $z$ direction is parallel to [001]. The $y$ axis forms the righthanded completion.

\begin{tabular}{|c|c|c|c|c|c|}
\hline Orientation & & $m_{x}\left(\mu_{B}\right)$ & $m_{y}\left(\mu_{B}\right)$ & $m_{z}\left(\mu_{B}\right)$ & $\phi_{\mathrm{DMI}}\left(^{\circ}\right)$ \\
\hline \multirow[t]{2}{*}{ Along $x\left(0^{\circ}\right)$} & $\boldsymbol{m}_{A}^{\mathrm{FM}}$ & 0.0246 & 0.000 & 0.0000 & 0.334 \\
\hline & $m_{A}^{\mathrm{AFM}}$ & 0.0000 & -4.210 & 0.0378 & \\
\hline \multirow[t]{2}{*}{ Along $y\left(90^{\circ}\right)$} & $\boldsymbol{m}_{A}^{\mathrm{FM}}$ & 0.000 & 0.0238 & 0.0039 & 0.324 \\
\hline & $\boldsymbol{m}_{A}^{\mathrm{AFI}}$ & 4.209 & 0.0000 & 0.0000 & \\
\hline \multirow[t]{2}{*}{ Along $116.9^{\circ}$} & $\boldsymbol{m}_{A}^{\mathrm{FN}}$ & -0.0098 & 0.0192 & 0.0000 & 0.294 \\
\hline & $\boldsymbol{m}_{A}^{\mathrm{AFM}}$ & 3.7533 & 1.9057 & -0.0002 & \\
\hline \multirow[t]{2}{*}{ Along $134.7^{\circ}$} & $\boldsymbol{m}_{A}^{\mathrm{FM}}$ & -0.0155 & 0.0156 & -0.0033 & 0.299 \\
\hline & $\boldsymbol{m}_{A}^{\mathrm{AFM}}$ & 2.9877 & 2.9652 & 0.0138 & \\
\hline \multirow[t]{2}{*}{ Along $160.2^{\circ}$} & $\boldsymbol{m}_{A}^{\mathrm{FM}}$ & -0.0218 & 0.0078 & -0.0010 & 0.315 \\
\hline & $\boldsymbol{m}_{A}^{\mathrm{AFM}}$ & 1.4262 & 3.9604 & -0.0141 & \\
\hline
\end{tabular}

resonant x-ray results of Beutier et al. [30]. However, for $\alpha-\mathrm{Fe}_{2} \mathrm{O}_{3}$ no such complementary $\mathrm{x}$-ray study is available so far. Thus, we performed density functional theory $a b$ initio quantum calculations for both compounds in order to add an independent DMI sign verification by an additional method. Nowadays, with continuously increasing computing power, DFT calculations have become an important and versatile tool to address scientific questions. Especially for complex magnetic structures, these simulations can be used in addition to the classical experiment to gain a deeper understanding of the underlying interactions and the observed effects.

We used the QUANTUM ESPRESSO $[57,58]$ DFT package to simulate the expected magnetization vectors in $\mathrm{MnCO}_{3}$ and $\alpha-\mathrm{Fe}_{2} \mathrm{O}_{3}$. The DFT calculations have been performed with the full-relativistic Perdew-Zunger norm-conserving pseudopotentials taking into account the spin-orbit interaction which is crucial for noncollinear magnetization. We used the local-spin-density approximation $(\operatorname{LSDA}+U)$ and the pseudopotentials from the web site of the THEOS Group at EPFL [59]: Mn.rel-pz-n-nc.UPF, C.rel-pznc.UPF, and O.rel-pz-nc.UPF for $\mathrm{MnCO}_{3}$ and Fe.rel-pz-
n-nc.UPF and O.rel-pz-nc.UPF for $\alpha-\mathrm{Fe}_{2} \mathrm{O}_{3}$. These pseudopotentials have been chosen simply because they provide rather fast convergence of the DFT calculations at our desktop workstation. The $6 \times 6 \times 6$ Monkhorst-Pack mesh [60] was used for both crystals: the wave function energy cutoff 50 (80) Ry, the charge-density energy cutoff 300 (500) Ry for $\mathrm{MnCO}_{3}\left(\alpha-\mathrm{Fe}_{2} \mathrm{O}_{3}\right)$.

In the previous PND studies on $\mathrm{WFM} \mathrm{NiF}_{2}$ [32] or $\mathrm{Gd}_{2} \mathrm{CuO}_{4}$ [33] the canting of the moments of the transition metal away from the main AFM axis could be attributed to some extent to crystal field effects of the coordinating polyhedra of neighboring anions. In the materials studied here, the rotation by the crystal field would be in the same direction as that of its coordinating polyhedron of ligands and would cause a magnetic anisotropy in the $a b$ plane. In order to clarify this, we performed QUANTUM ESPRESSO calculations of the expected magnetization vectors for different in-plane orientations of the WFM moment in $\mathrm{MnCO}_{3}$. The results are listed in Table III. The DMI angle shows very small deviations from its average value of $\phi_{\text {DMI }}=0.31(2)^{\circ}$. There is also practically no evidence for any anisotropy in the total energy with an average value of $E_{\text {tot }}=-290.38941099$ (1) Ry per unit cell and in the single-atom magnetization of $\left|\boldsymbol{m}_{i}\right|=4.21 \mu_{B}$. Thus, the observed canting is caused by the DMI rather than the crystal field, as it was assumed in Refs. [32,33].

By defining the WFM moment along the crystal [110] direction in $\mathrm{MnCO}_{3}$ and the [120] direction in $\alpha-\mathrm{Fe}_{2} \mathrm{O}_{3}$, thus almost perfectly along the field direction of the corresponding experiment, we could reproduce well the observed sign for $\boldsymbol{m}_{A}^{\mathrm{AFM}}$ and the DMI in both compounds as shown in Table IV. The simulated AFM moment for $\mathrm{MnCO}_{3}$ agrees perfectly with the measured one, whereas for $\alpha-\mathrm{Fe}_{2} \mathrm{O}_{3}$ it is slightly smaller. The clearly larger experimental $\boldsymbol{m}_{A}^{\mathrm{FM}}$ value of $\mathrm{MnCO}_{3}$ consists of the intrinsic WFM moment aligned by the field (around $0.03 \mu_{B}$ [43]) and an additional field-induced magnetic moment. The simulation in contrast does not consider the induced magnetic moment, explaining the observed difference.

TABLE IV. Magnetization vectors from DFT ab initio calculations for $\mathrm{MnCO}_{3}$ and $\alpha-\mathrm{Fe}_{2} \mathrm{O}_{3}$ compared to the experimental results from the PND data refinements (Tables I and II). For the simulations, the WFM moment was aligned along the crystal [110] direction in $\mathrm{MnCO}_{3}$ and the [120] direction in $\alpha-\mathrm{Fe}_{2} \mathrm{O}_{3}$, whereas for the experiment this direction is given by the applied magnetic field. The orthogonal $x y z$ directions of the magnetization are defined as $x$ parallel to the respective field, $y$ in the $a b$ plane, and $z$ as the right-handed completion (see Figs. 3 and 4).

\begin{tabular}{|c|c|c|c|c|c|c|c|c|}
\hline \multirow[b]{2}{*}{ Compound } & \multicolumn{4}{|c|}{ Calculation } & \multicolumn{4}{|c|}{ Experiment } \\
\hline & & $m_{x}\left(\mu_{B}\right)$ & $m_{y}\left(\mu_{B}\right)$ & $m_{z}\left(\mu_{B}\right)$ & & $m_{x}\left(\mu_{B}\right)$ & $m_{y}\left(\mu_{B}\right)$ & $m_{z}\left(\mu_{B}\right)$ \\
\hline \multirow[t]{2}{*}{$\mathrm{MnCO}_{3}$} & $\boldsymbol{m}_{4}^{\mathrm{FM}}$ & 0.0246 & 0 & 0 & $\boldsymbol{m}_{4}^{\mathrm{FM}}$ & $0.156(8)$ & 0 & 0 \\
\hline & $\boldsymbol{m}_{A}^{\mathrm{AFM}}$ & 0 & -4.210 & 0.0378 & $m_{4}^{\mathrm{A} M}$ & 0 & $-4.21(5)$ & $0.13(4)$ \\
\hline \multirow[t]{2}{*}{$\alpha-\mathrm{Fe}_{2} \mathrm{O}_{3}$} & $\boldsymbol{m}_{A}^{\mathrm{FM}}$ & 0.0054 & 0 & 0 & $\boldsymbol{m}_{A}^{\mathrm{FM}}$ & $0.006(7)$ & 0 & 0 \\
\hline & $\boldsymbol{m}_{A}^{\mathrm{AFM}}$ & 0 & -3.597 & 0.0009 & $\boldsymbol{m}_{A}^{\mathrm{AFM}}$ & 0 & $-3.69(6)$ & $-0.18(3)$ \\
\hline
\end{tabular}




\section{FURTHER EXAMPLES OF APPLICATION IN OTHER SYMMETRIES}

The symmetry analysis of the DMI vector presented in Sec. III and the PND method utilized in Sec. IV to experimentally determine the DMI sign in the two title compounds are generally valid and can be applied to any zero propagation WFM material. To emphasize this, we have analogously determined the DMI sign in two further compounds of different crystal systems.

The first compound is $\mathrm{HoFeO}_{3}$, a magnetocaloric material with orthorombic space group Pbnm, showing weak ferromagnetism in the high temperature $\Gamma_{4}$ phase, which is determined by Fe-Fe interactions, and in the low temperature $\Gamma_{2}$ phase, which is dominated by Ho-Ho interactions [61]. Applying the symmetry restrictions on the DMI vector presented in Sec. III A to the $\Gamma_{4}$ phase of $\mathrm{HoFeO}_{3}$, we found a restriction of the DMI vector normal to the [001] direction for the interplane and normal to the [100] direction for the intraplane interaction of iron atoms. For the $\Gamma_{2}$ phase, only the $z$ component of the DMI vector for the intraplane interaction of holmium atoms contributes by symmetry. Analyzing the experimentally determined magnetic moment directions of Chatterji et al. [34], we found a positive sign for the $y$ component of the interplane and intraplane $\mathrm{Fe}-\mathrm{Fe}$ DMI vector in the $\Gamma_{4}$ phase and a positive $z$ component of the intraplane Ho-Ho DMI vector in the $\Gamma_{2}$ phase. A more detailed analysis is given in the SM, Sec. VII A [40].

The second compound is $\mathrm{Ba}_{2} \mathrm{CoGe}_{2} \mathrm{O}_{7}$, an unconvential multiferroic material with tetragonal, noncentrosymmetric space group $P \overline{4} 2_{1} m$ [62]. It is weak ferromagnetically ordered below $T_{N} \approx 6.7 \mathrm{~K}$ with the AFM and WFM moments bound to the $a b$ plane [63]. Only the $z$ component of the DMI vector contributes to a WFM moment as determined by symmetry averaging, utilizing the restrictions given in Sec. III A. To determine the sign of $D_{z}$ in $\mathrm{Ba}_{2} \mathrm{CoGe}_{2} \mathrm{O}_{7}$, we have performed a PND study on a cylindrical high-quality $\mathrm{Ba}_{2} \mathrm{CoGe}_{2} \mathrm{O}_{7}$ single crystal at the VIP diffractometer at the Orphée reactor of LLB (Saclay, France). Analyzing the results of our PND experiment with the procedure presented in Sec. IV, we found a negative sign of the $D_{z}$ component. More details to our experiment, the symmetry averaging and the DMI sign determination, can be found in the SM, Sec. VII B [40]. A comprehensive analysis of the results from our PND study on $\mathrm{Ba}_{2} \mathrm{CoGe}_{2} \mathrm{O}_{7}$ will be published elsewhere; here we address only the sign of the DMI in this interesting compound.

\section{CONCLUSION}

To summarize, we have established polarized neutron diffraction as a powerful method to reliably determine the sign of the bulk Dzyaloshinskii-Moriya interaction in weak ferromagnets (canted antiferromagnets) with zero propagation vector. We could demonstrate that this determination is possible by measuring the asymmetry between intensities with up and down polarized neutrons of a single suitable Bragg reflection. This is of great value, given that such a single FR measurement requires much less experimental effort, measurement time, and is straightforward to interpret compared to the recently applied $[29,30]$ resonant $\mathrm{x}$-ray scattering technique. It can be easily applied to any WFM material with zero propagation vector, even under extreme sample environment conditions or using highly absorbing or weakly scattering samples of different types of modern highly topical materials.

We first validated the method on $\mathrm{MnCO}_{3}$, for which the DMI sign had previously been determined with resonant $\mathrm{x}$-ray scattering [30]. Our results both on the sign of the DMI and the details of the magnetic moment canting values are in perfect agreement with Ref. [30] as well as with our additionally performed $a b$ initio calculations.

After this validation, we applied the method to the classical WFM $\alpha-\mathrm{Fe}_{2} \mathrm{O}_{3}$ that had inspired the development of the theory of the DMI as an explanation of WFM [1-4], but for which the long-standing doubts about the absolute direction of the DMI could not be solved so far [49]. We were able to determine for the first time the negative sign of the DMI in this prototypical material. By ab initio calculations, we could reproduce the experimentally determined sign. Our results on the AFM domain distribution under applied magnetic field in $\alpha-\mathrm{Fe}_{2} \mathrm{O}_{3}$ may be of additional importance for the recently discovered phenomenon of protection of magnetic bits by AFM vortex domains in thin film $\alpha-\mathrm{Fe}_{2} \mathrm{O}_{3}$ [35], moving this material into the focal point of active current research in the field of future computing technologies, due to the ultrafast picosecond switching dynamics of AFM domain walls in comparison to classical FM domains.

Moreover, we have provided a universally valid approach to determine symmetry restrictions on the DMI vector imposed by the nuclear structure and have demonstrated the generality of the here presented PND method by additionally determining the DMI sign in orthorhombic $\mathrm{HoFeO}_{3}$ and tetragonal, noncentrosymmetric $\mathrm{Ba}_{2} \mathrm{CoGe}_{2} \mathrm{O}_{7}$.

We anticipate that with the method presented here, it will be possible to quickly and routinely determine the sign of the DMI in many more compounds.

\section{ACKNOWLEDGMENTS}

We would like to thank A. Rogalev for lending us the single crystals of hematite and rhodochroside used in this study and Dr. G. Bihlmayer for the fruitful discussion. The work was supported under the Tasso Springer Fellowship Program and V.E. D. was supported by the Ministry of Science and Higher Education of the Russian Federation within the State assignment FSRC "Crystallography and Photonics" RAS. The instrument POLI is operated by 
RWTH Aachen University and FZ Jülich (Jülich-Aachen Research Alliance JARA).

\section{APPENDIX: POLARIZED NEUTRON DIFFRACTION}

For PND with an incoming neutron polarization $\boldsymbol{P}$ parallel to the external magnetic field $\boldsymbol{H}$, the scattering cross section $\sigma_{q}$, which is proportional to the measured intensity $I$ of the diffracted beam, is given for a mixed nuclear-magnetic Bragg reflection by [64]

$$
\begin{aligned}
\sigma_{\boldsymbol{q}}(\boldsymbol{P})= & \underbrace{N N^{*}}_{\text {nuclear }}+\underbrace{\boldsymbol{M}_{\perp \boldsymbol{q}} \boldsymbol{M}_{\perp q}^{*}}_{\text {magnetic }} \\
& +\boldsymbol{P}(\underbrace{2 \operatorname{Re}\left[N \boldsymbol{M}_{\perp \boldsymbol{q}}^{*}\right]}_{\text {interference }}-\underbrace{i\left(\boldsymbol{M}_{\perp \boldsymbol{q}} \times \boldsymbol{M}_{\perp \boldsymbol{q}}^{*}\right)}_{\text {chiral }}),
\end{aligned}
$$

with $N$ and $\boldsymbol{M}$ the nuclear and magnetic structure factors and

$$
M_{\perp q}=\hat{\boldsymbol{q}} \times(\boldsymbol{M} \times \hat{\boldsymbol{q}})
$$

the part perpendicular to the scattering vector $\boldsymbol{q}$. The nuclear structure factor can be written as

$$
N=\sum_{j} b_{j} e^{-i \boldsymbol{q} \cdot \boldsymbol{r}_{j}},
$$

with the sum over all atoms $j$ in the unit cell at positions $\boldsymbol{r}_{j}$ and coherent mean scattering length $b_{j}$. Here and below, some positive factors (such as the Debye-Waller factor and conversion constants) are omitted, since they are well known and do not influence the determined sign of the DMI. The magnetic structure factor can be written in Bohr approximation as

$$
\boldsymbol{M}=\sum_{j} f_{j}(\boldsymbol{q}) \boldsymbol{m}_{j} e^{-i \boldsymbol{q} \cdot \boldsymbol{r}_{j}},
$$

where $f_{j}(\boldsymbol{q})$ is the magnetic form factor and $\boldsymbol{m}_{j}$ the magnetic moment of atom $j$ in the unit cell [64]. An approximation for the magnetic form factor is given by Brown [65]. Since all compounds investigated in this article are centrosymmetric, $N$ and $\boldsymbol{M}$ are both real and, thus, there is no chiral contribution. This reduces the measured asymmetry $A$ of a typical PND measurement to

$$
A_{\boldsymbol{q}}(\boldsymbol{P})=\frac{\sigma_{\boldsymbol{q}}(\boldsymbol{P})-\sigma_{\boldsymbol{q}}(-\boldsymbol{P})}{\sigma_{\boldsymbol{q}}(\boldsymbol{P})+\sigma_{\boldsymbol{q}}(-\boldsymbol{P})}=\frac{2 \boldsymbol{P} N \boldsymbol{M}_{\perp q}}{N N+\boldsymbol{M}_{\perp \boldsymbol{q}} \boldsymbol{M}_{\perp \boldsymbol{q}}} .
$$

It is clear to see that the sign of the asymmetry in a PND measurement reflects the sign of the magnetic structure factor relative to the neutron polarization for a known nuclear structure factor, and thus allows us to determine the sign of the DMI.
[1] I. E. Dzyaloshinskii, Thermodynamical Theory of "Weak" Ferromagnetism in Antiferromagnetic Substances, Sov. Phys. JETP 5, 1259 (1957), http://www.jetp.ac.ru/cgi-bin/ e/index/e/5/6/p1259?a=list.

[2] I. E. Dzyaloshinskii, A Thermodynamic Theory of "Weak" Ferromagnetism of Antiferromagnetics, J. Phys. Chem. Solids 4, 241 (1958).

[3] T. Moriya, New Mechanism of Anisotropic Superexchange Interaction, Phys. Rev. Lett. 4, 228 (1960).

[4] T. Moriya, Anisotropic Superexchange Interaction and Weak Ferromagnetism, Phys. Rev. 120, 91 (1960).

[5] S.-W. Cheong and M. Mostovoy, Multiferroics: A Magnetic Twist for Ferroelectricity, Nat. Mater. 6, 13 (2007).

[6] I. A. Sergienko and E. Dagotto, Role of the DzyaloshinskiiMoriya Interaction in Multiferroic Perovskites, Phys. Rev. B 73, 094434 (2006).

[7] M. Shiranzaei, J. Fransson, H. Cheraghchi, and F. Parhizgar, Nonlinear Spin Susceptibility in Topological Insulators, Phys. Rev. B 97, 180402(R) (2018).

[8] R. Cheng, M. W. Daniels, J.-G. Zhu, and D. Xiao, Antiferromagnetic Spin Wave Field-Effect Transistor, Sci. Rep. 6, 24223 (2016).

[9] M. Bode, M. Heide, K. von Bergmann, P. Ferriani, S. Heinze, G. Bihlmayer, A. Kubetzka, O. Pietzsch, S. Blügel, and R. Wiesendanger, Chiral Magnetic Order at Surfaces Driven by Inversion Asymmetry, Nature (London) 447, 190 (2007).

[10] S. Heinze, K. von Bergmann, M. Menzel, J. Brede, A. Kubetzka, R. Wiesendanger, G. Bihlmayer, and S. Blügel, Spontaneous Atomic-Scale Magnetic Skyrmion Lattice in Two Dimensions, Nat. Phys. 7, 713 (2011).

[11] Y. Togawa, T. Koyama, K. Takayanagi, S. Mori, Y. Kousaka, J. Akimitsu, S. Nishihara, K. Inoue, A. S. Ovchinnikov, and J. Kishine, Chiral Magnetic Soliton Lattice on a Chiral Helimagnet, Phys. Rev. Lett. 108, 107202 (2012).

[12] C. D. Hu, Giving Directions, Nat. Phys. 10, 180 (2014).

[13] J. Cho, N.-H. Kim, S. K. Kang, H.-K. Hwang, J. Jung, H. J. M. Swagten, J.-S. Kim, and C.-Y. You, The Sign of the Interfacial Dzyaloshinskii-Moriya Interaction in Ultrathin Amorphous and Polycrystalline Magnetic Films, J. Phys. D 50, 425004 (2017).

[14] S. Emori, U. Bauer, S. M. Ahn, E. Martinez, and G. S. Beach, Current-Driven Dynamics of Chiral Ferromagnetic Domain Walls, Nat. Mater. 12, 611 (2013).

[15] J. Torrejon, J. Kim, J. Sinha, S. Mitani, M. Hayashi, M. Yamanouchi, and H. Ohno, Interface Control of the Magnetic Chirality in CoFeB/MgO Heterostructures with Heavy-Metal Underlayers, Nat. Commun. 5, 4655 (2014).

[16] S. S. P. Parkin, M. Hayashi, and L. Thomas, Magnetic Domain-Wall Racetrack Memory, Science 320, 190 (2008).

[17] U. K. Rößler, A. N. Bogdanov, and C. Pfleiderer, Spontaneous Skyrmion Ground States in Magnetic Metals, Nature (London) 442, 797 (2006).

[18] S. Mühlbauer, B. Binz, F. Jonietz, C. Pfleiderer, A. Rosch, A. Neubauer, R. Georgii, and P. Boni, Skyrmion Lattice in a Chiral Magnet, Science 323, 915 (2009).

[19] N. S. Kiselev, A. N. Bogdanov, R. Schäfer, and U. K. Röler, Chiral Skyrmions in Thin Magnetic Films: New Objects for 
Magnetic Storage Technologies?, J. Phys. D 44, 392001 (2011).

[20] J. Sampaio, V. Cros, S. Rohart, A. Thiaville, and A. Fert, Nucleation, Stability and Current-Induced Motion of Isolated Magnetic Skyrmions in Nanostructures, Nat. Nanotechnol. 8, 839 (2013).

[21] A. Fert, V. Cros, and J. Sampaio, Skyrmions on the Track, Nat. Nanotechnol. 8, 152 (2013).

[22] C. Moreau-Luchaire, C. Moutafis, N. Reyren, J. Sampaio, C. A. F. Vaz, N. Van Horne, K. Bouzehouane, K. Garcia, C. Deranlot, P. Warnicke, P. Wohlhüter, J.-M. George, M. Weigand, J. Raabe, V. Cros, and A. Fert, Additive Interfacial Chiral Interaction in Multilayers for Stabilization of Small Individual Skyrmions at Room Temperature, Nat. Nanotechnol. 11, 444 (2016).

[23] X. Zhang, Y. Zhou, and M. Ezawa, Magnetic BilayerSkyrmions without Skyrmion Hall Effect, Nat. Commun. 7, 10293 (2016).

[24] K.-W. Kim, K.-W. Moon, N. Kerber, J. Nothhelfer, and K. Everschor-Sitte, Asymmetric Skyrmion Hall Effect in Systems with a Hybrid Dzyaloshinskii-Moriya Interaction, Phys. Rev. B 97, 224427 (2018).

[25] M. Belmeguenai, M. S. Gabor, Y. Roussigné, T. Petrisor, R. B. Mos, A. Stashkevich, S. M. Chérif, and C. Tiusan, Interfacial Dzyaloshinskii-Moriya Interaction Sign in $\mathrm{Ir} / \mathrm{Co}_{2} \mathrm{FeAl}$ Systems Investigated by Brillouin Light Scattering, Phys. Rev. B 97, 054425 (2018).

[26] S. V. Grigoriev, N. M. Potapova, S.-A. Siegfried, V. A. Dyadkin, E. V. Moskvin, V. Dmitriev, D. Menzel, C. D. Dewhurst, D. Chernyshov, R. A. Sadykov, L. N. Fomicheva, and A. V. Tsvyashchenko, Chiral Properties of Structure and Magnetism in $\mathrm{Mn}_{1-x} \mathrm{Fe}_{x} \mathrm{Ge}$ Compounds: When the Left and the Right are Fighting, Who Wins?, Phys. Rev. Lett. 110, 207201 (2013).

[27] S.-A. Siegfried, E. V. Altynbaev, N. M. Chubova, V. Dyadkin, D. Chernyshov, E. V. Moskvin, D. Menzel, A. Heinemann, A. Schreyer, and S. V. Grigoriev, Controlling the Dzyaloshinskii-Moriya Interaction to Alter the Chiral Link between Structure and Magnetism for $\mathrm{Fe}_{1-x} \mathrm{Co}_{x} \mathrm{Si}$, Phys. Rev. B 91, 184406 (2015).

[28] Y. Kousaka, T. Koyama, K. Ohishi, K. Kakurai, V. Hutanu, H. Ohsumi, T. Arima, A. Tokuda, M. Suzuki, N. Kawamura, A. Nakao, T. Hanashima, J. Suzuki, J. Campo, Y. Miyamoto, A. Sera, K. Inoue, and J. Akimitsu, Monochiral Helimagnetism in Homochiral Crystals of $\mathrm{CsCuCl}_{3}$, Phys. Rev. Mater. 1, 071402(R) (2017).

[29] V.E. Dmitrienko, E. N. Ovchinnikova, S.P. Collins, G. Nisbet, G. Beutier, Y. O. Kvashnin, V. V. Mazurenko, A. I. Lichtenstein, and M. I. Katsnelson, Measuring the Dzyaloshinskii-Moriya Interaction in a Weak Ferromagnet, Nat. Phys. 10, 202 (2014).

[30] G. Beutier, S. P. Collins, O. V. Dimitrova, V. E. Dmitrienko, M. I. Katsnelson, Y. O. Kvashnin, A. I. Lichtenstein, V. V. Mazurenko, A. G. A. Nisbet, E. N. Ovchinnikova, and D. Pincini, Band Filling Control of the Dzyaloshinskii-Moriya Interaction in Weakly Ferromagnetic Insulators, Phys. Rev. Lett. 119, 167201 (2017).

[31] D. Pincini, F. Fabrizi, G. Beutier, G. Nisbet, H. Elnaggar, V. E. Dmitrienko, M. I. Katsnelson, Y. O. Kvashnin, A. I. Lichtenstein, V. V. Mazurenko, E. N. Ovchinnikova, O. V.
Dimitrova, and S. P. Collins, Role of the Orbital Moment in a Series of Isostructural Weak Ferromagnets, Phys. Rev. B 98, 104424 (2018).

[32] P. J. Brown and J. B. Forsyth, A Neutron Diffraction Study of Weak Ferromagnetism in Nickel Fluoride, J. Phys. C 14, 5171 (1981).

[33] P. J. Brown and T. Chatterji, Weak Ferromagnetism and Magnetic Phase Transitions in $\mathrm{Gd}_{2} \mathrm{CuO}_{4}$, Phys. Rev. B 84, 054426 (2011).

[34] T. Chatterji, A. Stunault, and P. J. Brown, Single Crystal Polarized Neutron Diffraction Study of the Magnetic Structure of $\mathrm{HoFeO}_{3}$, J. Phys. Condens. Matter 29, 385802 (2017).

[35] F. P. Chmiel, N. W. Price, R. D. Johnson, A. D. Lamirand, J. Schad, G. V. D. Laan, D. T. Harris, J. Irwin, M. S. Rzchowski, C. Eom, and P. G. Radaelli, Observation of Magnetic Vortex Pairs at Room Temperature in a Planar $\alpha-\mathrm{Fe}_{2} \mathrm{O}_{3} /$ Co Heterostructure, Nat. Mater. 17, 581 (2018).

[36] V. Hutanu, M. Meven, E. Lelièvre-Berna, and G. Heger, POLI-HEiDi: The New Polarised Neutron Diffractometer at the Hot Source (SR9) at the FRM II-Project Status, J. Phys. B 404, 2633 (2009).

[37] V. Hutanu, POLI: Polarised Hot Neutron Diffractometer, J. Large-Scale Res. Facil. 1, A16 (2015).

[38] H. Thoma, W. Luberstetter, J. Peters, and V. Hutanu, Polarized Neutron Diffraction Using a Novel High- $T_{c}$ Superconducting Magnet on the Single-Crystal Diffractometer POLI at MLZ, J. Appl. Crystallogr. 51, 17 (2018).

[39] H. Thoma, H. Deng, G. Roth, and V. Hutanu, Setup for Polarized Neutron Diffraction Using a High-T Superconducting Magnet on the Instrument POLI at MLZ and Its Applications, J. Phys. Conf. Ser. 1316, 012016 (2019).

[40] See Supplemental Material at http://link.aps.org/ supplemental/10.1103/PhysRevX.11.011060 for a more detailed description of the crystallographic structure of the investigated compounds, for a proof that the DMI vector transforms as an axial vector, for mathematical calculations and for additional supporting experimental studies including the DMI sign determination in two further compounds.

[41] A. H. Hill, F. Jiao, P. G. Bruce, A. Harrison, W. Kockelmann, and C. Ritter, Neutron Diffraction Study of Mesoporous and Bulk Hematite, $\alpha-\mathrm{Fe}_{2} \mathrm{O}_{3}$, Chem. Mater. 20, 4891 (2008).

[42] A. S. Borovik-Romanov and M. P. Orlova, Magnetic Properties of Cobalt and Manganese Carbonates, Sov. Phys. JETP 4, 531 (1957), http://www.jetp.ac.ru/cgi-bin/e/index/ e/4/4/p531?a=list.

[43] A. S. Borovik-Romanov, Investigation of Weak Ferromagnetism in the $\mathrm{MnCO}_{3}$ Single Crystal, Sov. Phys. JETP 9 , 539 (1959), http://www.jetp.ac.ru/cgi-bin/e/index/e/9/3/ p539?a=list.

[44] V. E. Dmitrienko, E. N. Ovchinnikova, J. Kokubun, and K. Ishida, Dzyaloshinskii-Moriya Interaction: How to Measure Its Sign in Weak Ferromagnets?, JETP Lett. 92, 383 (2010).

[45] R. A. Alikhanov, Neutron Diffraction Investigation of the Antiferromagnetism of the Carbonates of Manganese, and Iron, Sov. Phys. JETP 9, 1204 (1959), http://www.jetp.ac .ru/cgi-bin/e/index/e/9/6/p1204?a=list. 
[46] P. J. Brown and J. B. Forsyth, The Spatial Distribution of Ferromagnetic Moment in $\mathrm{MnCO}_{3}$, Proc. Phys. Soc. 92 , 125 (1967).

[47] C. G. Shull, W. A. Strauser, and E. O. Wollan, Neutron Diffraction by Paramagnetic and Antiferromagnetic Substances, Phys. Rev. 83, 333 (1951).

[48] S. N. Klausen, K. Lefmann, P.-A. Lindgård, L. T. Kuhn, C. R. H. Bahl, C. Frandsen, S. Mørup, B. Roessli, N. Cavadini, and C. Niedermayer, Magnetic Anisotropy and Quantized Spin Waves in Hematite Nanoparticles, Phys. Rev. B 70, 214411 (2004).

[49] R. Nathans, S. J. Pickart, H. A. Alperin, and P. J. Brown, Polarized Neutron Study of Hematite, Phys. Rev. 136, A1641 (1964).

[50] J. Hönigschmid and G. Will, Feldinduzierte Domänenstrukturen in Hämatit $\left(\alpha-\mathrm{Fe}_{2} \mathrm{O}_{3}\right)$, J. Magn. Magn. Mater. 4, 220 (1977).

[51] N. Qureshi, Mag2Pol: A Program for the Analysis of Spherical Neutron Polarimetry, Flipping Ratio and Integrated Intensity Data, J. Appl. Crystallogr. 52, 175 (2019).

[52] V. Petíček, M. Dušek, and L. Palatinus, Crystallographic Computing System JANA2006: General Features, Z. Kristallogr. 229, 345 (2014).

[53] J. C. Matthewman, P. Thompson, and P. J. Brown, The Cambridge Crystallography Subroutine Library, J. Appl. Crystallogr. 15, 167 (1982).

[54] G. J. Redhammer, A. Senyshyn, G. Tippelt, and G. Roth, Magnetic Spin Structure of Pyroxene-Type $\mathrm{MnGeO}_{3}$, J. Phys. Condens. Matter 23, 254202 (2011).

[55] G. Shirane, S. J. Pickart, and Y. Ishikawa, Neutron Diffraction Study of Antiferromagnetic $\mathrm{MnTiO}_{3}$ and $\mathrm{NiTiO}_{3}, \mathrm{~J}$. Phys. Soc. Jpn. 14, 1352 (1959).
[56] V. Baron, J. Gutzmer, H. Rundlöf, and R. Tellgren, Neutron Powder Diffraction Study of Mn-Bearing Hematite, $\alpha-\mathrm{Fe}_{2-x} \mathrm{Mn}_{x} \mathrm{O}_{3}$, in the Range $0 \leq x \leq 0.176$, Solid State Sci. 7, 753 (2005).

[57] P. Giannozzi et al., QUANTUM ESPRESSO: A Modular and Open-Source Software Project for Quantum Simulations of Materials, J. Phys. Condens. Matter 21, 395502 (2009).

[58] P. Giannozzi et al., Advanced Capabilities for Materials modelling with QUANTUM ESPRESSO, J. Phys. Condens. Matter 29, 465901 (2017).

[59] EPFL-THEOS Group, Standard Solid-State Pseudopotentials (SSSP), http://theossrv1.epfl.ch/Main/Pseudopotentials, accessed April 27, 2020.

[60] H. J. Monkhorst and J. D. Pack, Special Points for BrillouinZone Integrations, Phys. Rev. B 13, 5188 (1976).

[61] T. Chatterji, M. Meven, and P. J. Brown, Temperature Evolution of Magnetic Structure of $\mathrm{HoFeO}_{3}$ by Single Crystal Neutron Diffraction, AIP Adv. 7, 045106 (2017).

[62] H. Murakawa, Y. Onose, S. Miyahara, N. Furukawa, and Y. Tokura, Comprehensive Study of the Ferroelectricity Induced by the Spin-Dependent $d-p$ Hybridization Mechanism in $\mathrm{Ba}_{2} X \mathrm{Ge}_{2} \mathrm{O}_{7}(X=\mathrm{Mn}, C o$, and $C u)$, Phys. Rev. $\mathrm{B}$ 85, 174106 (2012).

[63] H. T. Yi, Y. J. Choi, S. Lee, and S.-W. Cheong, Multiferroicity in the Square-Lattice Antiferromagnet of $\mathrm{Ba}_{2} \mathrm{CoGe}_{2} \mathrm{O}_{7}$, Appl. Phys. Lett. 92, 212904 (2008).

[64] G. L. Squires, Introduction to the Theory of Thermal Neutron Scattering, 3rd ed. (Cambridge University Press, Cambridge, England, 2012).

[65] P. J. Brown, Magnetic Form Factors, in International Tables for Crystallography Vol. C: Mathematical, Physical and Chemical Tables (Springer Netherlands, Dordrecht, 2006), Chap. 4, pp. 454-461. 